Received Date : 24-Jan-2016

Revised Date :-29Feb-2016

Accepted Date : 01-Mar-2016

Article ype : Oiginal Article

Revised manuscript for Indoor Air, February 2016

\title{
Evaporation and dispersion of respiratory droplets from coughing
}

Li Liu ${ }^{1}$, Jianjian Wei ${ }^{1 *}$, Yuguo $\mathrm{Li}^{1}$, Andrew Ooi ${ }^{2}$

${ }^{1}$ Department of Mechanical Engineering, the University of Hong Kong, Pokfulam Road,

Hong Kong SAR, China

${ }^{2}$ Department of Mechanical Engineering, the University of Melbourne, Parkville, Melbourne,

Australia

*Corresponding author:

Jianjian Wei

Department of Mechanical Engineering, The University of Hong Kong

Pokfulam Road, Hong Kong SAR, China

Tel: (852) 63590238

E-mail: wei.jianjian.88@gmail.com

This is the author manuscript accepted for publication and has undergone full peer review but has not been through the copyediting, typesetting, pagination and proofreading process, which may lead to differences between this version and the Version of Record. Please cite this article as doi: 10.1111/ina.12297

This article is protected by copyright. All rights reserved 
Potential referees:

- Professor Mark Nicas, University of California Berkeley

- Professor Donald Milton, Maryland Institute for Applied Environmental Health; Email: dmilton@umd.edu

Word count of abstract: 198

Word count of text: 6912

\title{
Evaporation and dispersion of respiratory droplets from coughing
}

\begin{abstract}
Understanding how respiratory droplets become droplet nuclei and their dispersion is essential for understanding the mechanisms and control of disease transmission via droplet-borne and airborne routes. A theoretical model was developed to estimate the size of droplet nuclei and their dispersion as a function of the ambient humidity and droplet composition. The model-predicted dried droplet nuclei size was $32 \%$ of the original diameter, which agrees with the maximum residue size in the classic study by Duguid [Edinburg Med.
\end{abstract} $J ., 52,335-340(1946)]$ and the validation experiment in this study, but is smaller than the 50\% size predicted by Nicas et al. [J. Occup. Environ. Hyg., 2, 143-154 (2005)]. The droplet nuclei size at a relative humidity of $90 \%\left(25^{\circ} \mathrm{C}\right)$ could be $30 \%$ larger than the size of the same droplet at a relative humidity of less than $67.3 \%\left(25^{\circ} \mathrm{C}\right)$. The trajectories of respiratory droplets in a cough jet are significantly affected by turbulence, which promotes the wide dispersion of droplets. We found that medium-sized droplets (e.g., $60 \mu \mathrm{m}$ ) are more influenced by humidity than are smaller and larger droplets, while large droplets $(\geq 100 \mu \mathrm{m})$, whose travel is less influenced by humidity, quickly settle out of the jet.

Key words: Respiratory droplet; Droplet nuclei; Equilibrium; Droplet nuclei size; Evaporation; Dispersion. 


\section{Practical Implications}

Our study reveals the influence of indoor humidity, turbulence and the composition of respiratory droplets on the size and dispersion of droplet nuclei and their destination after being released. These findings may be useful both for investigating the mechanism of disease transmission via large droplets or airborne routes and for developing engineering control methods to prevent infection in hospitals and the community.

\section{Introduction}

Respiratory droplets or droplet nuclei in the indoor environment have been known to be carriers of potential pathogens since they were first identified by Wells (1934). At least two important questions remain to be resolved, i.e., how droplets become droplet nuclei during their dispersion process, and how evaporation and turbulence affect the dispersion distance of droplets. Evidence suggests that the size distribution and travel distances of droplet nuclei can significantly influence infection risk in the indoor environment (Xie et al., 2007).

A droplet nucleus is the final product of a respiratory droplet after being released into air for a sufficient time. Droplet nuclei are derived from respiratory droplets, initially produced by the atomization of human secretions along the airway (Hare, 1964). The chemical composition of secretions lining the surface of the human airway has been described by Potter et al. (1963), Dodds et al. (1997), Effros et al. (2002) and Schipper et al. (2007), among others. Sodium and potassium cations and chloride anion are the nonvolatile ionic solutes, and lactate and glycoprotein are the organic substances existing in exhaled condensates (Effros et al., 2002). The influence of the initial composition of respiratory droplets on droplet nuclei size is not well understood.

We regard the size of exhaled droplets just outside the mouth or nose as the initial size of the droplets, with the size of the droplet nuclei formed out of these droplets varying during the evaporation and dispersion process. A number of studies have determined the sizes of respiratory droplets and droplet nuclei, although significant inconsistencies exist. A microscope method was used by Duguid $(1945,1946)$ in which droplet initial sizes of 5-2000 $\mu \mathrm{m}$ and droplet nuclei sizes of 0.25-42 $\mu \mathrm{m}$ were measured. Loudon and Roberts (1967) found droplet initial sizes to be $10-1473 \mu \mathrm{m}$ and droplet nuclei sizes to be $3-10 \mu \mathrm{m}$. Very small 
droplet nuclei sizes, mostly $<1 \mu \mathrm{m}$, were found by Fairchild and Stampfer (1987) and Papineni and Rosenthal (1997) using an optical particle counter. Fennelly et al. (2004) first measured the size distribution of infectious aerosols produced by patients with pulmonary tuberculosis in the size range of 2.1-3.3 $\mu \mathrm{m}$. Yang et al. (2007) used an aerodynamic particle sizer and scanning mobility particle sizer and found that the droplet nuclei sizes were 0.58-5.42 $\mu \mathrm{m}$. The interferometric Mie imaging technique was used by Chao et al. (2009), who found the mean initial droplet size to be $13.5 \mu \mathrm{m}$ for coughing and $16.0 \mu \mathrm{m}$ for speaking. Xie et al. (2009) used glass slides, a microscope and an aerosol spectrometer and determined the peak (mode) initial size for large droplets to be 50-75 $\mu \mathrm{m}$. Using the data on droplet composition from Effros et al. (2002), Nicas et al. (2005) were probably the first to suggest that a respiratory droplet could ultimately have a larger residue size in humid air than in dry air. They estimated that the diameter of the droplet nuclei was about half that of the initial droplet diameter. In their analysis, a high concentration of glycoprotein $(76 \pm 18 \mathrm{~g} / \mathrm{L})$ was used, resulting in a relatively larger size than our estimate in this paper. The concentration of glycoprotein in saliva is only about $2 \mathrm{~g} / \mathrm{L}$ according to Dodds et al. (1997) and Schipper et al. (2007), and the concentration of organic polymers including proteins, lipid and carbohydrate in pulmonary mucus is about $2.8 \mathrm{~g} / \mathrm{L}$ according to Potter et al. (1963). With this set of data and the estimation method of Nicas et al. (2005), the diameter of droplet nuclei is only about a quarter of the initial diameter. Thus, significant discrepancies exist in the literature in estimating the size of droplet nuclei.

The drying out of droplets is well studied (Ranz and Marshall, 1952; Kukkonen et al., 1989, among others). Fang et al. (2006) presented a study of the effect of deoxyribonucleic acid (DNA) suspended as nanoparticles on the evaporation of a deposited droplet. The existence of DNA lowered the evaporation rate considerably after the majority of water was lost. DNA can form shell- or gel-like solids during evaporation. However, a theoretical model of the behavior of suspended DNA inside a droplet does not exist. Xie et al. (2007) simplified respiratory droplets as pure water droplets and estimated their travel distances with various initial sizes and mean flow fields. Large droplets (initial diameter greater than $60 \mu \mathrm{m}$ ) were found to be deposited on the floor at a distance of $1.5 \mathrm{~m}$ from the mouth, when the droplets were initially expelled at a velocity of $10 \mathrm{~m} / \mathrm{s}$ at a $2 \mathrm{~m}$ height from the floor. It would be useful to establish whether the travel distance of a respiratory droplet is influenced by residue size, relative humidity $(\mathrm{RH})$ and turbulence. 


\section{Methodology}

Our simple developed model includes an estimate of the droplet nuclei size, the drying-out process and the movement of droplets and droplet nuclei as affected by turbulence and the cough jet. The composition of respiratory droplets varies depending on their point of origin. We define the initial conditions at the mouth or nose when the droplets are about to be expelled. The initial size is denoted by $d_{i}$, the mass fraction of sodium chloride by $c_{i}$, the volume ratio of insoluble solids by $\Phi_{i}$, and the ambient air temperature $T$ and RH are given.

\section{A simple model of droplet nuclei size $d_{r}$}

The aqueous part of a respiratory droplet is assumed to be sodium chloride solution in our model for the purpose of simplification. For some organic substances, e.g., glycoproteins that are strongly hygroscopic, their effects are simplified to be represented by the solutes because their amounts are not conclusive based on the current literature. The initial concentration is given as $150 \mathrm{mM}$, similar to the concentration in plasma. The solid part of a respiratory droplet including all of the suspended mucous organics and potential pathogens is assumed to be insoluble solids. The density of the solids is assumed to be $2000 \mathrm{~kg} / \mathrm{m}^{3}$, and the specific heat capacity is $1000 \mathrm{~kJ} /\left(\mathrm{kg} \cdot{ }^{\circ} \mathrm{C}\right)$. The initial solid volume ratio is $1.8 \%$ according to Duguid (1946).

The sodium chloride and potassium chloride dissolved in respiratory droplets have very strong hygroscopicity, and they tend to absorb vapor from the ambient air. In humid air, the droplets are composed of an aqueous solution instead of desiccated particles (Tang, 1999). Pruppacher and Klett (1997) investigated and reviewed the effects of insoluble solids suspended in liquid droplets in equilibrium.

In dry air, when droplets evaporate to their droplet nuclei sizes, the insoluble solids and nonvolatile solutes form a crust that is assumed to be spherical, and the droplet nuclei size is the size of this crust.

$$
d_{c}=\left(\frac{\Phi_{\mathrm{i}}^{*}}{\Phi_{\max }}\right)^{\frac{1}{3}} d_{i}
$$

where $d_{c}$ is the crust diameter, $\Phi_{\mathrm{i}}^{*}=\Phi_{\mathrm{i}}+\bar{\rho}_{l} c_{i} / \rho_{N a C l}, \overline{\rho_{l}}$ is the mean density of the initial droplet, $\rho_{\mathrm{NaCl}}$ is the density of crystallized sodium chloride, and $\Phi_{\max }$ is 0.5236 , the maximum solid volume ratio accounting for the porosity of the solid residue (Sirignano, 1999).

This article is protected by copyright. All rights reserved 
When the droplets evaporate to their droplet nuclei sizes in humid air, the phase equilibrium is assumed to occur on the droplet surface, and then the droplet nuclei size is the equilibrium size. To determine this size, we choose the droplet and its surrounding environment as a system at a constant temperature $T$ and a constant total pressure $p$. For a given temperature $T$, there is a saturation vapor pressure $p_{s a t}$, and $p_{\infty}$ is the partial pressure of vapor in ambient air. The partial pressure of vapor on the droplet surface is denoted by $p_{s}$. Generally, $p_{\infty}<p_{s}<p_{s a t}$, as illustrated in Figure 1a.

We consider two effects, i.e., the Kelvin effect, in which the curvature of the droplet surface requires a higher partial vapor pressure on the droplet surface to maintain its equilibrium size, and the solute effect, in which a lower partial vapor pressure is required by the nonvolatile solutes to maintain equilibrium. For a solution droplet, a general equation

$$
\ln \frac{p_{s}}{p_{s a t}}=\ln a_{w}+\frac{4 M_{w} \sigma_{L V}}{\rho_{w} R T_{\infty} d_{e}}
$$

is applied to relate the equilibrium diameter and the partial pressure of vapor. In Equation (2), $R$ is the gas constant. $M_{w}$ and $\rho_{w}$ are the molecular mass and density of water, respectively, and $\sigma_{L V}$ is the surface tension of the liquid-vapor interface. If the droplet is pure water, the water activity $a_{w}=1$, and then the vapor pressure $p_{s}$ is larger than $p_{s a t}$, because the right-hand side of Equation (2) is positive. When equilibrium occurs at a smaller equilibrium diameter $d_{e}, p_{s}$ needs to have a larger value in the equation, in accordance with the Kelvin effect. For a solution droplet, $a_{w}<1$, and if $a_{w}$ is quite small, the required partial pressure $p_{s}$ could be equal to $p_{\infty}$. Here, we simply assume $p_{s}=p_{\infty}$, although the equilibrium diameter $d_{e}$ is underestimated by this assumption.

Based on the theory of Pruppacher and Klett (1997), the water activity $a_{w}$ could be expressed as follows

$$
a_{w}=\exp \left(-v \Psi_{\text {salt }} \frac{n_{s}}{n_{w}}\right)
$$

where $v$ is the total number of ions into which a salt molecule dissociates. For $\mathrm{NaCl}, v=2$. $\Psi_{\text {salt }}$ is the practical osmotic coefficient as a function of the salt concentration, with $\Psi_{\text {salt }}=$ 1.2 here, and $n_{s}$ and $n_{w}$ are the numbers of moles of the solute and water, respectively.

Substituting Equation (3) into (2) and expanding the number of moles of the solute and water $n_{s}$ and $n_{w}$, we obtain 


$$
\ln \frac{p_{\infty}}{p_{\text {sat }}}=\ln R H=\frac{4 M_{w} \sigma_{L V}}{\rho_{w} R T_{\infty} d_{e}}-\frac{v \Psi_{\text {salt }} M_{w} c_{i}\left(1-\Phi_{i}\right)}{M_{\text {salt }}\left(1-c_{i}\right)} \frac{1}{\left(\frac{d_{e}}{d_{i}}\right)^{3}-\Phi_{i}}
$$

At the threshold RH (TRH), the equilibrium size equals the crust size, $d_{e}=d_{c}$. Using Equation (1) and (4), we obtain

$$
T R H^{*}=\exp \left[\frac{4 M_{w} \sigma_{L V}}{\rho_{w} R T_{\infty} d_{i}}\left(\frac{\Phi_{\max }}{\Phi_{i}}\right)^{\frac{1}{3}}-\frac{v \Psi_{\text {salt }} M_{w} c_{i}\left(1-\Phi_{i}\right)}{M_{\text {salt }}\left(1-c_{i}\right)} \frac{\Phi_{\max }}{\Phi_{i}-\Phi_{i} \Phi_{\max }}\right]
$$

When RH is greater than the TRH, the equilibrium size is larger than the crust size. Then, the droplet nuclei size of a droplet $\left(d_{r}\right)$ becomes

$$
d_{r}= \begin{cases}d_{c}=f\left(d_{i}, \Phi_{i}\right) & \mathrm{RH} \leq \mathrm{TRH} \\ d_{e}=f\left(d_{i}, \Phi_{i}, c_{i}, T_{\infty}, \mathrm{RH}\right) & \mathrm{RH}>\mathrm{TRH}\end{cases}
$$

where the diameter $d_{c}$ for dry air is determined using Equation (1), and $d_{e}$ for humid air is determined using Equation (4).

\section{Droplet drying-out process}

When a respiratory droplet is released into air, heat and mass transfer occur simultaneously at the droplet surface. We assume that the sodium chloride does not crystallize during evaporation. We also assume that the droplet remains spherical, and the temperature of the droplet is uniform but different from the ambient temperature.

The partial pressure of vapor on the droplet surface, $p_{s}$, is assumed to be equal to the saturation vapor pressure, $p_{s a t}$. The differential $\frac{d m_{d}}{d t}$ is the evaporation rate, $d_{s}$ is the droplet diameter as a function of time, $p$ is the atmospheric pressure of air, $T_{\infty}$ is the temperature far from droplets, $D_{\infty}$ is the diffusion coefficient of vapor, $\mathrm{Sh}=1+0.38 \mathrm{Re}^{1 / 2} \mathrm{Sc}^{1 / 3}$ (the Sherwood number), and $p_{s}$ and $p_{\infty}$ are, respectively, the partial vapor pressure on the droplet surface and distant from the droplet. Considering the Stefan flow and temperature dependence of diffusion coefficients, Kukkonen et al. (1989) gave the evaporation rate as:

$$
\frac{d m_{d}}{d t}=\frac{2 \pi p d_{s} M_{w} D_{\infty} C_{T} S h}{R T_{\infty}} \ln \left(\frac{p-p_{s}}{p-p_{\infty}}\right)
$$

where $C_{T}=\frac{T_{\infty}-T_{p}}{T_{\infty}^{\lambda-1}} \frac{2-\lambda}{T_{\infty}^{2-\lambda}-T_{p}^{2-\lambda}}(\lambda$ is a constant between 1.6 and 2$)$ is a correction factor because of the temperature dependence of the diffusion coefficient.

This article is protected by copyright. All rights reserved 
Heat balance at the droplet surface requires that the enthalpy change of the droplet be composed of heat conduction, evaporation and thermal radiation. Here, we omit thermal radiation.

$$
\left(m_{l} C_{l}+m_{p} C_{p}\right) \frac{d T_{s}}{d t}=\pi d_{s}^{2} K_{g} \frac{T_{\infty}-T_{s}}{r_{s}} N u+L_{v} \frac{d m_{d}}{d t}
$$

where $m_{l}$ and $C_{l}$ are the respective mass and the specific heat of the solution. The specific heat of the solution is assumed to be that of pure water, and $m_{p}$ and $C_{p}$ are the respective mass and specific heat of the insoluble particles, $K_{g}$ is the thermal conductivity of gas, and $L_{v}$ is the latent heat of vaporization.

Various forces act on the droplet in the air including gravitational, drag, pressure gradient, virtual mass, Basset, thermophoretic, Brownian, Saffman's lift forces, and possible electric forces. The pressure gradient, virtual mass and Basset forces were found to be two orders of magnitude smaller than the drag force for droplet movement in indoor air (Zhao et al., 2004). To determine the droplet velocity $V_{d}$, we assume that there are only gravitational, buoyancy and drag forces on the droplet. Applying Newton's second law yields the following equation:

$$
m_{d} \frac{d \boldsymbol{V}_{\boldsymbol{d}}}{d t}=m_{d} \boldsymbol{g}\left(1-\frac{\rho_{d}}{\rho_{g}}\right)-C_{d} \pi d_{s}{ }^{2} \frac{\rho_{g}\left|\boldsymbol{V}_{\boldsymbol{d}}-\boldsymbol{V}_{\boldsymbol{g}}\right|\left(\boldsymbol{V}_{\boldsymbol{d}}-\boldsymbol{V}_{\boldsymbol{g}}\right)}{8}
$$

where $C_{d}$ is the drag coefficient and $V_{g}$ is the air velocity. According to Liu et al. (1993),

$$
\begin{gathered}
R e=\frac{\rho_{g} d_{s}\left|V_{\boldsymbol{d}}-\boldsymbol{V}_{\boldsymbol{g}}\right|}{\mu} \\
C_{d}=\left\{\begin{array}{cc}
0.424 & R e>1000 \\
\frac{24}{R e}\left(1+\frac{1}{6} R e^{\frac{2}{3}}\right) & R e \leq 1000
\end{array}\right.
\end{gathered}
$$

The displacement of droplet $S_{d}$ is

$$
\frac{d \boldsymbol{S}_{\boldsymbol{d}}}{d t}=\boldsymbol{V}_{\boldsymbol{d}}
$$

\section{The cough jet}

Once expelled into the air, respiratory droplets interact with the exhaled airflow and also the ambient airflows. Coughing has probably been studied the most among all respiratory activities. Visualization of droplet trajectories by Bourouiba et al. (2015) revealed the wide spread of exhaled droplets by a violent cough. Here, we model the cough flow as a circular turbulent buoyant jet, and we suspend respiratory droplets into it. The airflow velocity along 
the jet is calculated, and it is used in Equations (8) and (11) to track the trajectory of a droplet. The effect of ambient turbulence is also evaluated. The jet model is presented in the Supporting Material.

A particle tracking model, i.e., the discrete random walk model first proposed by Gosman and Ioannides (1983), is used here to predict the effect of turbulence on particle dispersion. In the discrete random walk model, or "eddy lifetime" model, the fluctuating velocity components are discrete piecewise constant functions of time, and their random value is kept constant over an interval of time, which is the minimum of either the eddy lifetime or the transit time required for the particle to cross the eddy.

$$
\begin{aligned}
& t_{\text {int }}=\min \left(t_{e}, t_{r}\right) \\
& t_{e}=2 t_{L}=2 C_{L} \frac{k}{\varepsilon} \\
& t_{r}=-\tau \ln \left(1.0-\frac{l_{E}}{\tau\left|V_{g}-V_{d}\right|}\right)
\end{aligned}
$$

where $t_{L}=C_{L} \frac{k}{\varepsilon}$ is the Lagrangian integral time scale, $C_{L} \approx 0.15$ for the $k-\varepsilon$ model and its variants, $\tau$ is the particle relaxation time defined as $\tau=\frac{4}{3} \rho_{p} d_{p} /\left(\rho_{g} C_{D}\left|V_{g}-V_{d}\right|\right)$, $\left|V_{g}-V_{d}\right|$ is the magnitude of the relative velocity at the start of the interaction, $l_{E}=C_{\mu}^{3 / 4} k^{3 / 2} / \varepsilon$ is the eddy length scale, and $C_{\mu}=0.09$. Instead of using computational fluid dynamics (CFD), the $k$ and $\varepsilon$ are calculated using the jet model in the Supporting Material.

\section{A simple droplet evaporation experiment}

A simple but effective experiment was conducted to measure saliva droplet evaporation. One droplet of saliva was released by a pipette onto the surface of a Teflon printed slide with an initial contact angle $\theta_{c}$ larger than $90^{\circ}$ (Figure 2a and 2b). Humidity was controlled inside the measuring chamber. The mass loss of the droplet was recorded by an analytical balance. Note that the evaporation process of a droplet suspended in the air must be different from that of a deposited droplet. The evaporation rate of a droplet deposited on a solid surface depends on 
the contact angle (Chandra et al., 1996 and Birdi et al., 1989).

We tested droplets of pure water and droplets human saliva with an initial volume of $10 \mu \mathrm{L}$ $\left(d_{i} \approx 1337 \mu \mathrm{m}\right)$. All of the saliva tested was collected from one healthy male. The mass loss of the droplet at RH of $64 \%, 84 \%$ and $96 \%\left(25^{\circ} \mathrm{C}\right)$ was recorded by an analytical balance with an accuracy of $0.1 \mathrm{mg}$ [Model AUW220, Shimadzu, Japan]. The ambient temperature and RH were measured by a Humidity Temperature Meter [Model 311, Center Technology, Taiwan]. The ambient humidity was controlled by a humidifier [Model 403c, Yuyue Medical Equipment, China].

\section{Results}

\section{Agreement between simple droplet nuclei formation model and experimental results}

The droplet nuclei size of a droplet of $\mathrm{NaCl}$ solution or of $\mathrm{KCl}$ solution as a function of $\mathrm{RH}$ was used to validate the equilibrium size by comparing it to the experimental data from Tang (1999), as shown in Figure 3a. The mass fractions of $\mathrm{NaCl}$ and $\mathrm{KCl}$ solutes were $7.7 \%$ and $10 \%$, respectively, under equilibrium at a $\mathrm{RH}=95 \%$; no insoluble solids were contained in the droplets. The trend of predicted droplet nuclei size with $\mathrm{RH}$ follows the experimental data well, and the maximum error is less than $10 \%$.

The predicted size of the droplet nuclei is mainly determined by the volume ratio of insoluble solids. Using the same initial volume ratio of solids, $1.8 \%$ as in Duguid (1945), the predicted peak of the crust size distribution is the same as the measured peak of the droplet nuclei size distribution (Figure 3b).

Figure 4 shows the results of our validation experiments. All tests were conducted in a stable indoor environment. The ambient air temperature was about $25^{\circ} \mathrm{C}$. The airflow velocity close to the deposited droplet was less than $0.1 \mathrm{~m} / \mathrm{s}$. For a droplet with $c_{i}=0.9 \%$ and $\Phi_{i}=1.8 \%$, the TRH is $67 \%$ according to Equation (6). The mass loss of droplets at RH 96\%, $84 \%$ and $64 \%$ was measured. In Figure $4 \mathrm{a}, \mathrm{RH}$ is $96 \%$, and we predicted a faster evaporation than the measured result. In the experiment, we observed that the mass of both the water droplets and saliva droplets roughly maintained their initial masses, i.e., the phase equilibrium was reached at the droplet surface. Hence, the saliva droplet nuclei size in this instance is larger than our predicted result of $0.7 d_{i}$. When the air was less humid, a difference between the shape evolution of a pure water droplet and a saliva droplet was observed in the measurement. 
The contact angle of the pure water droplet was maintained throughout the measurement, whereas that of the saliva droplet decreased sharply during the measurement. At the end of the measurement, the shape of the saliva droplet on the Teflon surface was flat. Choi and Kim (2009) explained this phenomenon as the contact angle of the deposited protein droplet decreasing during evaporation due to protein adsorption on the substrate surface, and the pinning effect. We estimated the equivalent droplet nuclei size from its mass. In Figure 4b, the predicted saliva droplet nuclei size is $0.35 d_{i}$, and the measured equivalent nuclei size is $0.34 \pm 0.03 d_{i}$. The mass of the droplet residue is about $3 \%$ of the initial droplet mass of about $10 \mathrm{mg}$, so the precision of the balance $(0.1 \mathrm{mg})$ is sizable, and this might result in an error in the mass measurement. The predicted evaporation rates of water droplets and saliva droplets are almost equal to each other in the first 50 minutes. After 50 minutes, however, the saliva droplet evaporates more slowly than the water droplet as the solution effect becomes stronger with the increase in concentration. However, we predicted faster evaporation rates both in Figure $4 \mathrm{~b}$ and 4c, probably because the droplet shape changed in the measurement. Due to the curvature effect, vapor molecules leave liquid phase faster from a curved surface than a flat surface. This effect lowered the evaporation rate when the contact angle was decreasing. In fact, droplet evaporation is most sensitive to the change of $\mathrm{RH}$ at high $\mathrm{RH}$ conditions. In Figure 4c, the droplet nuclei size is predicted to be the crust size, $0.32 d_{i}$, at RH $64 \%$, less than the TRH of $67 \%$. The measured equivalent residue size is $0.31 d_{i}[+114 \%$, $-92 \%]$. The mass loss by time shows an almost-linear relation for both the experimental results and the predicted results at RH 64\%. The predicted results match the evaporation rates of the water droplet, but still there is some discrepancy for the saliva droplet. The difference in results between our model and the experiments could be due to the assumptions about droplet composition and to the assumption that the evaporation properties of the deposited droplets and suspended droplets are similar.

\section{Effect of initial solid volume ratio and salt solution on droplet nuclei sizes}

The effects of initial solid volume fraction $\Phi_{i}$ and initial salt mass fraction $c_{i}$ on the TRH and droplet nuclei size are calculated and shown in Figure 5a and 5b. The TRH increases with solid volume fraction $\Phi_{i}$ and decreases with initial salt mass fraction $c$ in the range: $1 \%<\Phi_{i}$ $<3 \%, 0.1 \%<c_{i}<0.9 \%$. When solid volume fraction $\Phi_{i}$ is $0.6 \%$ or $1.00 \%$ and initial salt mass fraction $c_{i}$ is $0.9 \%$, the TRH could be as low as the approximate crystallization RH. The hygroscopicity of the solute is revealed in Figure 5a. Increasing the salt mass fraction $c_{i}$ decreases the TRH and increases the equilibrium size. In Figure $5 b$, the solid volume ratio $\Phi_{i}$ 
directly influences the crust size. Duguid (1946) measured the residue sizes of six droplets suspended on fine glass fibers and found that the residue size varied from one-fifth to one-third that of the original. The predicted dimensionless diameter $d \approx 0.32$ when $\Phi_{i}$ is $1.8 \%$ matches the maximum residue size in Duguid's tests and is larger than the average value of 0.25 estimated by Duguid (1946). However, our predicted dimensionless diameter is not as large as the estimation by Nicas et al. (2005) until the RH is no less than $97 \%$. A quite high concentration of glycoprotein was adopted by Nicas et al. (2005), which occupied about 7.6\% of the total volume initially.

\section{Effects of RH on droplet trajectories}

The trajectories of droplets in a cough jet with different sizes for two RH (0\% and $90 \%)$ are shown in Figure 6, when the initial salt mass fraction is $0.9 \%$ and the initial solid volume ratio is $1.8 \%$. Hence, the TRH is about $67.3 \%$. The peak velocity is $10 \mathrm{~m} / \mathrm{s}$, and the mouth has an equivalent diameter of $2 \mathrm{~cm}$ for a typical cough jet (Gupta et al., 2009). Because the ambient air is assumed to be stagnant, the distance that one droplet can travel is dominated by how long the droplet can remain before it escapes from the jet. When turbulence is excluded from the jet, droplets with an initial diameter larger than $80 \mu \mathrm{m}$ will always be deposited on the floor from an initial height of $2 \mathrm{~m}$ (Figure 6a and 6b). The distance that these large droplets can travel away from the mouth is around $1 \mathrm{~m}$. The droplet with an initial diameter of $60 \mu \mathrm{m}$ has a totally different destination when the RH changes. It can reach the end of the jet, about $4 \mathrm{~m}$, with a nuclei size of $0.32 d_{i}$ at a $\mathrm{RH}$ of $0 \%$, whereas it can only travel a distance of $1.85 \mathrm{~m}$ at a RH of $90 \%$ due to the larger droplet nuclei size of $0.43 d_{i}$.

The distance an expelled droplet can travel depends significantly on humidity and droplet nuclei size. Figure 7 presents the ratio of the maximum horizontal distances a droplet can reach when $\mathrm{RH}$ is $0 \%$ and $90 \%$. This ratio becomes unity for small droplets, $d_{i}<35 \mu \mathrm{m}$, and large droplets, $d_{i}>70 \mu \mathrm{m}$, when the initial speed is $5 \mathrm{~m} / \mathrm{s}$. The range increases to $45 \mu \mathrm{m}$ and $80 \mu \mathrm{m}$, respectively, when the initial speed is $10 \mathrm{~m} / \mathrm{s}$. These droplets either travel to the end of the jet or fall onto the floor. For droplets with a medium initial size $(50 \mu \mathrm{m})$, the maximum distance ratio can reach about 3 (initial speed $=5 \mathrm{~m} / \mathrm{s}$ ). This suggests that at this initial speed, a droplet with initial size of $50 \mu \mathrm{m}$ can travel 2 times further horizontally under very dry conditions $(\mathrm{RH}=0 \%)$ than that at a $\mathrm{RH}=90 \%$. Droplets travel to quite different destinations under different humidities or equivalently different droplet compositions. 
The dispersion of exhaled droplets differs with droplet size and humidity. Droplet dispersion profiles of $20 \mu \mathrm{m}, 60 \mu \mathrm{m}$ and $100 \mu \mathrm{m}$ droplets in a steady turbulent cough jet are shown in Figure 8. The effect of turbulence on droplet dispersion is significant (compared with Figure 6). Small droplets $(20 \mu \mathrm{m})$ follow the airflow closely and are dispersed almost in the whole jet region (marked by the top-hat jet half-width, which corresponds to the visible jet boundary), whereas large droplets $(100 \mu \mathrm{m})$ seem not much affected by the airflow. They have ballistic trajectories and deposit not long after their release. Medium-sized droplets (60 $\mu \mathrm{m})$ disperse widely, and no deposition occurs before $x=2 \mathrm{~m}$.

The dispersion of droplets with an initial diameter of $60 \mu \mathrm{m}$ is shown when the observer is located 0.8, 1.2, 1.6 m upstream (Figure 9). The droplet evaporates more slowly and the droplet nuclei size is larger when RH is $90 \%$. It takes 0.2 seconds for a droplet to travel $0.8 \mathrm{~m}$ in an exhalation jet in which the centerline rises by less than $0.01 \mathrm{~m}$ because the jet initial momentum is dominant over the buoyancy effect. The exhaled droplet still maintains almost the same height as that at the initial position. At $1.2 \mathrm{~m}$, droplets exhaled into humid air when RH is $90 \%$ start to escape from the jet. Meanwhile the droplets exhaled into dry air have just approached the boundary of the jet at $1.2 \mathrm{~m}$ and start to escape from the jet at $1.6 \mathrm{~m}$. In addition, droplets in high $\mathrm{RH}$ are less dispersed due to their larger size and to inertia in the same spanwise location. It should be noted that $0 \%$ and $90 \%$ are two extreme possibilities of $\mathrm{RH}$ and that the actual trajectories would fall somewhere in between. Ambient air is not generally stagnant in practice. Airflows induced by a ventilation system, human plume or human movement can sometimes change the destinations of exhaled droplets, especially the fine droplet nuclei.

\section{Discussion}

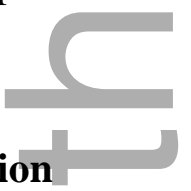

In the infection control literature, a diameter of $5 \mu \mathrm{m}$ or $10 \mu \mathrm{m}$ is often cited as the threshold droplet size (Brankston, et al., 2007). However, it is not clear whether the droplet size refers to the initial size at the mouth/nose release point, the interim size at the point of large droplet exposure (within 1 or $1.5 \mathrm{~m}$ from the source) or the final size of the eventually formed droplet nuclei. Our results clearly show that these three sizes have different characteristics. Droplets of both the interim size and final size vary according to environmental conditions and their initial size. However, the initial size can be considered to be a consistent parameter, which 
does not change with the environment. Therefore, our suggestion is to use the initial size of the exhaled droplet in the definition of large droplet transmission.

This study suggests that droplet nuclei of less than $10 \mu \mathrm{m}$ are involved in the airborne transmission route. Droplet transmission can involve any size of droplet, but it mostly occurs at close proximity and involves droplets larger than $10 \mu \mathrm{m}$ in terms of initial size. The threshold size of large droplet fall-out is $60-80 \mu \mathrm{m}$. Some of the exhaled large droplets (initial sizes of less than $60 \mu \mathrm{m}$ ) involved in droplet transmission can still remain suspended in the air, but for a much smaller period of time than the air-change time scale of one hour in a typical room.

The importance of the initial salt and solid concentrations of the droplet is clearly shown in our analysis. We simplified the composition of exhaled droplets and assumed the same composition for all sizes of droplets and for all people, which may not be realistic. For example, Potter et al. (1963) reported that the total concentration of sodium cation and potassium cation in the mucus from a healthy person was 0.38 times lower than that from cystic fibrosis patients, whereas the concentration of insoluble solids in the mucus from cystic fibrosis patients was about 2.64 times higher than that from healthy persons. With today's chemical analysis capability, it should be possible to carry out a detailed chemical analysis of the composition of droplet nuclei and/or respiratory droplets for people of different ages and health status, during different stages of a respiratory infection, and for different diseases. Presently, such data is not available.

This study also reveals that the residue size of respiratory droplets is a function of indoor humidity. In dry conditions, droplets evaporate quickly and can remain suspended in the air for a long time, thus increasing the exposure risk. There is evidence showing that indoor RH influences the transmission of respiratory diseases. Lowen et al. (2007) measured the transmission efficiency of influenza virus using the guinea pig as a model host and found it to be close to $100 \%$ at low $\mathrm{RH}$ at both $5^{\circ} \mathrm{C}$ and $20^{\circ} \mathrm{C}$. At $20^{\circ} \mathrm{C}$, the transmission efficiency diminished at a RH of about $50 \%$ and then rose again at a $\mathrm{RH}$ of about $70 \%$. The transmission efficiency then decreased to zero when the RH was above $80 \%$. Transmission was more efficient at $5^{\circ} \mathrm{C}$ than at $20^{\circ} \mathrm{C}$, but the infection rate was reduced to $50 \%$ at a $\mathrm{RH}$ of $70 \%$ and maintained a value above zero at a $\mathrm{RH}$ of $80 \%$. The importance of $\mathrm{RH}$ may be relevant when considering the infection risk in aircraft cabins, where the RH can be as low as $10 \%$ (Hunt et al., 1995). Thus, dry air could be key to the spread of infection in aircraft. 


\section{Limitations of the present study}

There is at least one major shortcoming in the present droplet nuclei model in relation to the assumption of thermodynamic equilibrium. The partial pressure at the droplet surface is actually higher than that in ambient air, rather than equal as assumed in our model. This equilibrium is meta-stable, and crystallization may occur any time during droplet evaporation, especially with the suspended particles. This means that ultimately, the droplet nuclei size will be the size of the crust. The crust may break up into smaller pieces in the air or on impact with a surface. Further, the hygroscopicity of the glycoproteins might have a significant effect on droplet evaporation, and crystallization process is not considered in our droplet evaporation model; however, this is impossible to predict precisely due to the complexity of the droplet components. Although we used saliva droplets in our validation experiment, respiratory droplets originating from the trachea or the lung might have different compositions. In addition, the cough puff is simplified to be a steady jet with a constant injection velocity in the dispersion analysis; however, Bourouiba et al. (2015) revealed the existence of a leading vortex in the dynamic cough puff. The use of CFD simulation to model more realistic breathing cycles is considered in Liu et al. (submitted for publication).

\section{Conclusions}

We analyzed the process by which an exhaled droplet becomes a droplet nucleus using a simple physical model. In our model, an existing equilibrium theory was used to estimate the residue size of a respiratory droplet, and an evaporation theory and a non-isothermal jet model were adopted to simulate the evaporation and dispersion of the droplet in the cough airflow. The formation model of the droplet nuclei closely replicated the test results of a simple experiment. The effects of initial salt concentration, initial solid volume ratio and $\mathrm{RH}$ on droplet nuclei size, evaporation and dispersion were tested by numerical computations. Our predicted size of dried-out droplet nuclei is $32 \%$ of the original diameter, which agrees with the maximum residue size in the classic Duguid (1946) study, but is smaller than the $50 \%$ predicted by Nicas et al. (2005). The droplet nuclei maintain a larger size in humid air than that in dry air. The droplet nuclei size when RH is $90 \%$ could be $30 \%$ larger than that in dry air when the initial salt mass fraction is $0.9 \%$ and the initial solid volume ratio is $1.8 \%$.

Small droplets can reach their residue sizes and be transported; the distances traveled and the ultimate destinations are related to initial size, residue size and humidity. Turbulence in the This article is protected by copyright. All rights reserved 
cough jet prompts the wide dispersion of droplets. Medium-sized droplets $(60 \mu \mathrm{m})$ are more influenced by humidity, whereas large droplets quickly settle out of the jet and are less influenced by it. At $0 \% \mathrm{RH}$ ambient conditions, $60-\mu \mathrm{m}$ droplets would dry out and become droplet nuclei with a diameter of $19 \mu \mathrm{m}$, which follow the cough jet when turbulence is not a factor. However, under turbulent conditions, some of the droplet nuclei fall out of the jet due to wide dispersion. Such droplet nuclei are able to travel more than $4 \mathrm{~m}$. For 100- $\mu \mathrm{m}$ droplets, the mean travel distance is about $1 \mathrm{~m}$ at $0 \% \mathrm{RH}$.

\section{Acknowledgements}

We are grateful for the financial support received from the RGC (HKU7142/12) and NSFC (Grant numbers 51278440 and 51378415). We are also grateful to Professor William Nazaroff of the University of California at Berkeley for his first suggestion of the balance experiment of droplets for validation.

\section{References}

Birdi, K.S., Vu, D.T. and Winter, A. (1989) A study of the evaporation rates of small water drops placed on a solid surface, J. Phys. Chem., 93, $3702-3703$.

Bourouiba,L., Dehandschoewercker, E. and Bush, J.W.M. (2015) Violent expiratory events: on coughing and sneezing, J. Fluid Mech., 745, 537 - 563.

Brankston, G., Gitterman, L., Hirji, Z., Lemieux, C. and Gardam, M. (2007) Transmission of influenza A in human beings, Lancet Infect. Dis., 7, 257-265.

Chandra, S., Di Marzo, M., Qiao, Y.M. and Tartarini, P. (1996) Effect of liquid-solid contact angle on droplet evaporation, Fire Safety J., 27, 141-158.

Chao, C.Y.H., Wan, M.P., Morawska, L., Johnson, G.R., Ristovski, Z.D., Hargreaves, M., Mengersen, K., Corbett, S., Li, Y., Xie, X. and Katoshevski, D. (2009) Characterization of expiration air jets and droplet size distributions immediately at the mouth opening, $J$. Aerosol Sci., 40, 122-133.

Choi, C.H. and Kim, C.J. (2009) Droplet evaporation of pure water and protein solution on nanostructured superhydrophobic surfaces of varying heights, Langmuir, 25(13),

This article is protected by copyright. All rights reserved 
$7561-7567$.

Dodds, M.W., Johnson, D.A., Mobley, C.C. and Hattaway, K.M. (1997) Parotid saliva protein profiles in caries-free and caries active adults, Oral Surg. Oral Med. Oral Pathol. Oral Radiol. Endod., 83, 244-251.

Duguid, J.F. (1945) The numbers and the sites of origin of the droplets expelled during expiratory activities, Edinburg Med. J., 52, 335-340.

Duguid, J.F. (1946) The size and duration of air-carriage of respiratory droplets and droplet-nuclei, J. Hyg., 4, 471-480.

Effros, R.M., Wahlen, K., Bosbous, M., Castillo, D., Foss, B., Dunning, M., Gare, M., Lin, W. and Sun, F. (2002) Dilution of respiratory solutes in exhaled condensates, Am. J. Resp. Crit. Care. Med., 165, 663-669.

Fairchild, C.I. and Stampfer J.F. (1987) Particle concentration in exhaled breath, Am. Ind. Hyg. Assoc. J., 48, 948-949.

Fang, X., Li, B., Peterson, E., Seo, Y., Samuilov, V.A., Chen, Y., Sokolov, J.C., Shew, C. and Rafailovich M.H. (2006) Drying of DNA droplets, Langmuir, 22(14), 6308-6312.

Fennelly, K.P., Martyny, J.W., Kayte, E.F., Orme, I.M., Cave, D.M. and Heifets, L.B. (2004) Isolation of viable airborne Mycobacterium tuberculosis: a new method to study transmission, Am. J. Respir. Crit. Care Med., 169, 604-609.

Gosman, A.D. and Ioannides, E. (1983) Aspects of computer-simulation of liquid-fueled combustors. J. Energy, 7(6), 482-490.

Gupta, J.K., Lin, C.H. and Chen, Q. (2009) Flow dynamics and characterization of a cough. Indoor Air, 19(6): 517 - 525

Hare, R. (1964) The transmission of respiratory infections, Proc. Roy. Soc. Med., 57, $221-230$.

Hunt, E.H., Reid, D.H., Space, D.R. and Tilton, F.E. (1995) Commercial airliner environmental control system - engineering aspects of cabin air quality. In: Proceedings of the Annual Meeting of the Aerospace Medical Association, 1-8.

Kukkonen, J., Vesala, T. and Kulmala, M. (1989) The interdependence of evaporation and settling for airborne freely falling droplets, J. Aerosol. Sci., 20, 749-763.

Liu, A.B., Mather, D. and Reitz, R.D. (1993) Modeling the Effects of Drop Drag and Breakup This article is protected by copyright. All rights reserved 
on Fuel Sprays, SAE Technical Paper 930072.

Loudon, R.G. and Roberts, R.M. (1967) Relation between the airborne diameter of respiratory droplets and the diameter of the stains left after recovery, Nature, 213, 95-96.

Lowen, A.C, Mubareka, S., Steel, J. and Palese, P. (2007) Influenza virus transmission is dependent on relative humidity and temperature, PLoS Pathog., 3, 1470-1476.

Nicas, M., Nazaroff, W.W. and Hubbard, A. (2005) Toward understanding the risk of secondary airborne infection: emission of respirable pathogen, J. Occup. Environ. Hyg., 2, $143-154$.

Papineni, R.S. and Rosenthal, F.S. (1997) The size distribution of droplets in the exhaled breath of healthy human subjects, J. Aerosol Med., 10, 105-116.

Potter, J.L., Matthews, L.W., Lemm, J. and Spector, S. (1963) Human pulmonary secretions in health and disease, Am. Rev. Respir. Dis., 96, 83-87.

Pruppacher, H.R. and Klett, J.D. (1997) Microphysics of Clouds and Precipitation: Second Revised and Enlarged Edition with an Introduction to Cloud Chemistry and Cloud Electricity, Norwell, MA, Kluwer Academic Publishers.

Ranz, W. E. and Marshall, W. R. (1952) Evaporation from drops, Chem. Eng. Prog., 48, $141-146$ and $173-180$.

Schipper, R. G., Siletti, E. and Vingerhoeds, M. H. (2007) Saliva as research material: biochemical, physicochemical and practical aspects, Arch. Oral Biol., 52, 1114-1135.

Sirignano, W.A. (1999) Fluid Dynamics and Transport of Droplets and Sprays, Cambridge, Cambridge University Press.

Tang, I.N. (1999) Phase transformation and growth of hygroscopic aerosols, In: Spurny, K.R. (ed.) Aerosol Chemical Processes in the Environment, Boca Raton, FL, CRC Press LLC.

Wells, W.F. (1934) On air-borne infection. Study II. Droplets and droplet nuclei, Am. J. Hyg., 20, 611-618.

Xie, X., Li, Y., Chwang, A.T., Ho, P.L. and Seto, W.H. (2007) How far droplets can move in indoor environments - revising the Wells evaporation-falling curve, Indoor Air, 17, 211-225.

Xie, X., Li, Y., Sun, H.Q. and Liu, L. (2009) Exhaled droplets due to talking and coughing, J. R. Soc. Interface, 6, 703-714.

This article is protected by copyright. All rights reserved 
Yang, S., Lee, G.W.M., Chen, C.M., Wu, C.C. and Yu, K.P. (2007) The size and concentration of droplets generated by coughing in human subjects, J. Aerosol Med., 20, 484-494.

Zhao, B., Zhang, Y., Li, X., Yang, X. and Huang, D. (2004) Comparison of indoor aerosol particle concentration and deposition in different ventilated rooms by numerical method, Build. Environ., 39, 1-8.

\section{List of figures}
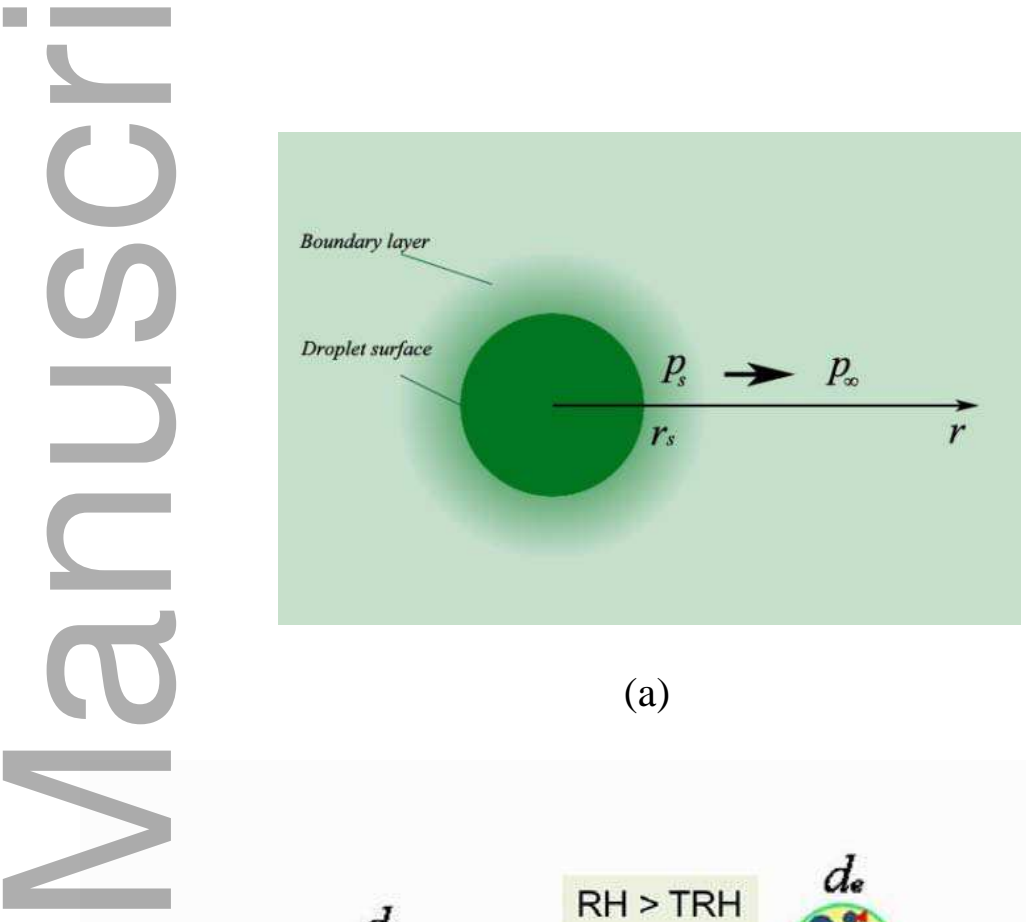

(a)
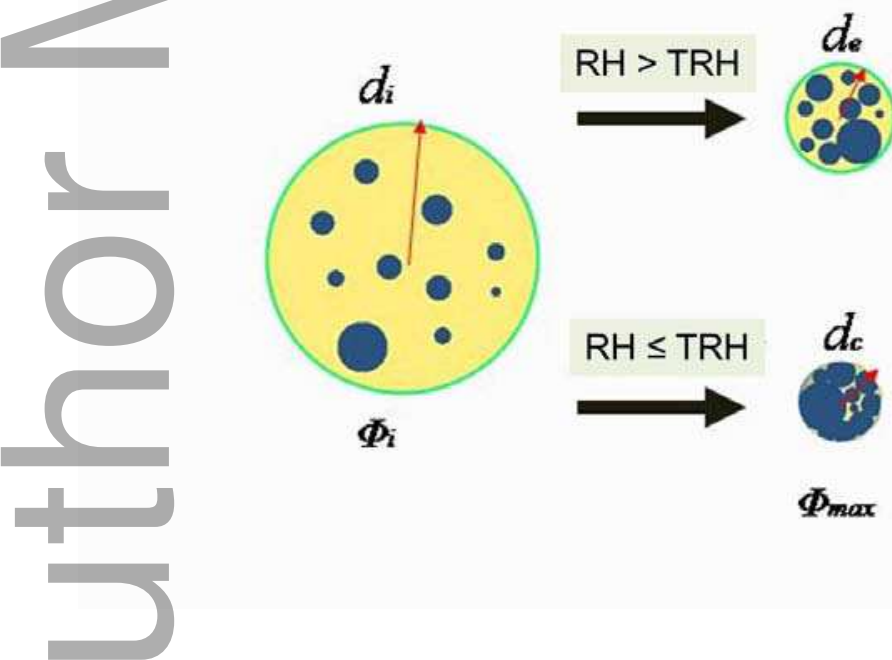

(b)

Figure 1. A simple droplet nuclei formation model. (a) Partial pressure distribution diagram. (b) The initial droplet forms a crust at low relative humidity $(\mathrm{RH})$ or maintains equilibrium at high RH. TRH, threshold relative humidity. 


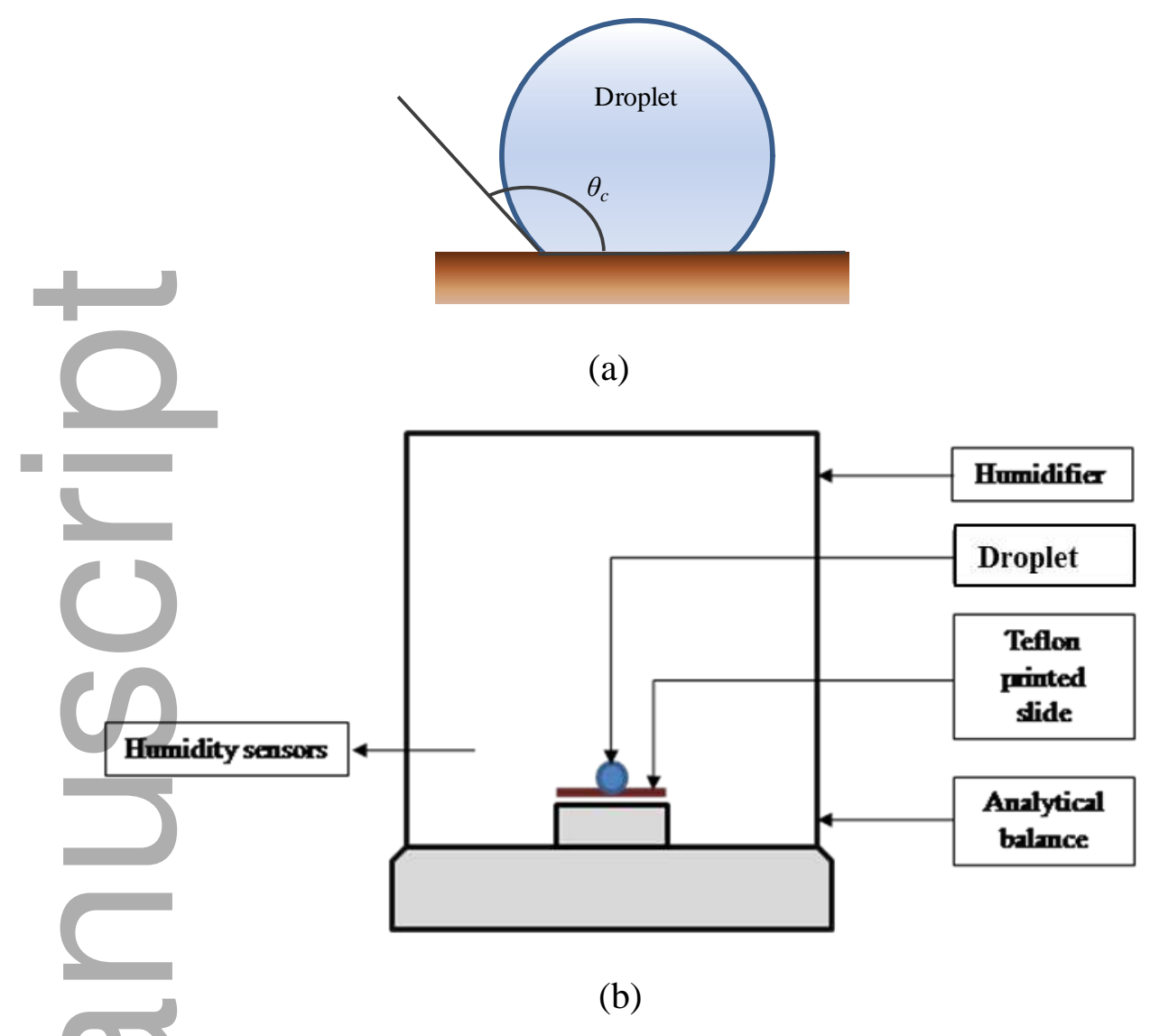

Figure 2. (a) Illustration of a droplet deposited upon a Teflon printed slide. (b) Schematic diagram of the test setup.

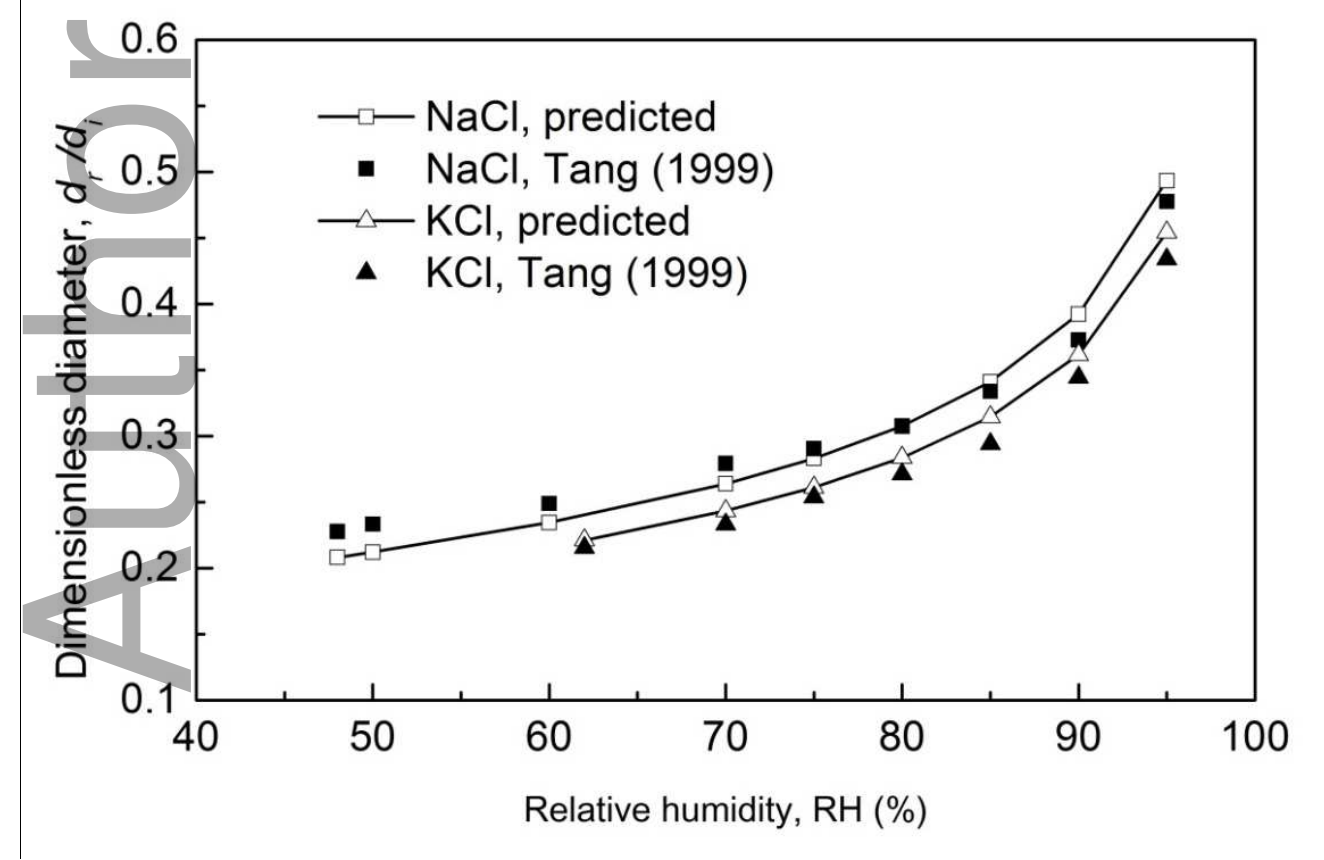

(a)

This article is protected by copyright. All rights reserved 


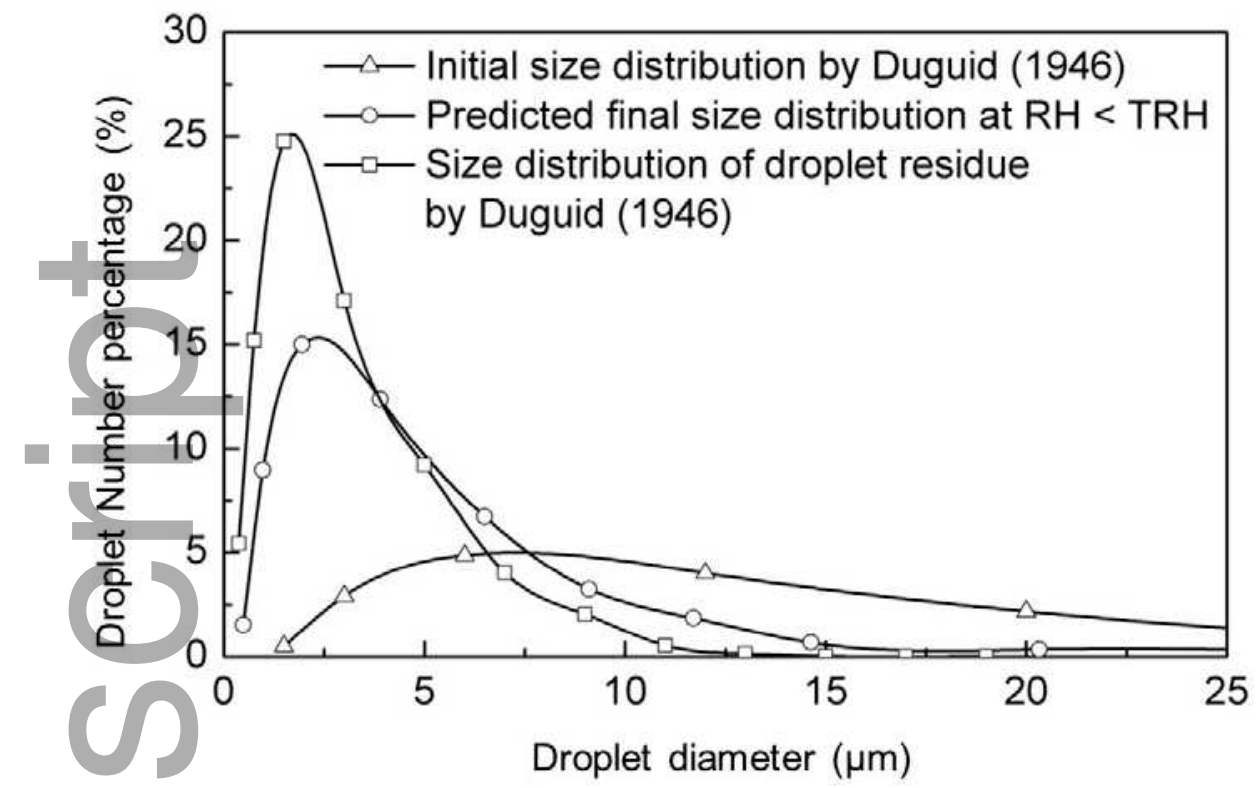

(b)

Figure 3. (a) Comparison of the predicted equilibrium size with varying RH using the present model and experimental results from Tang (1999). There are no insoluble solids in the experiment; the mass fractions of $\mathrm{NaCl}$ and $\mathrm{KCl}$ solutes are $7.7 \%$ and $10 \%$, respectively, under equilibrium at a $\mathrm{RH}=95 \%$. (b) Comparison of the crust size predicted using the present model and experimental results from Duguid (1945) when the initial insoluble volume ratio is $1.8 \%$, and there are no solutes. TRH, threshold relative humidity.

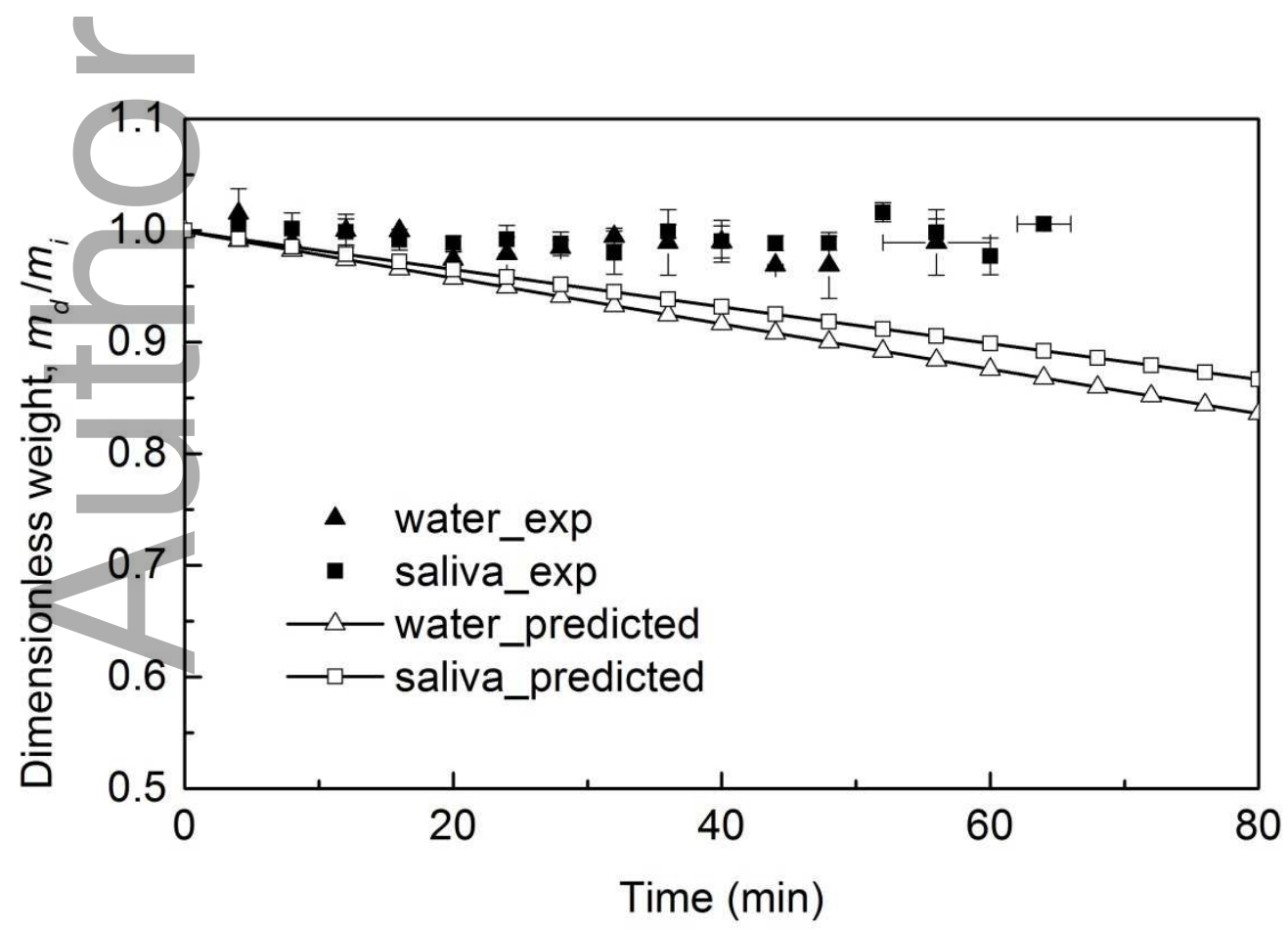

This article is protected by copyright. All rights reserved 
(a)

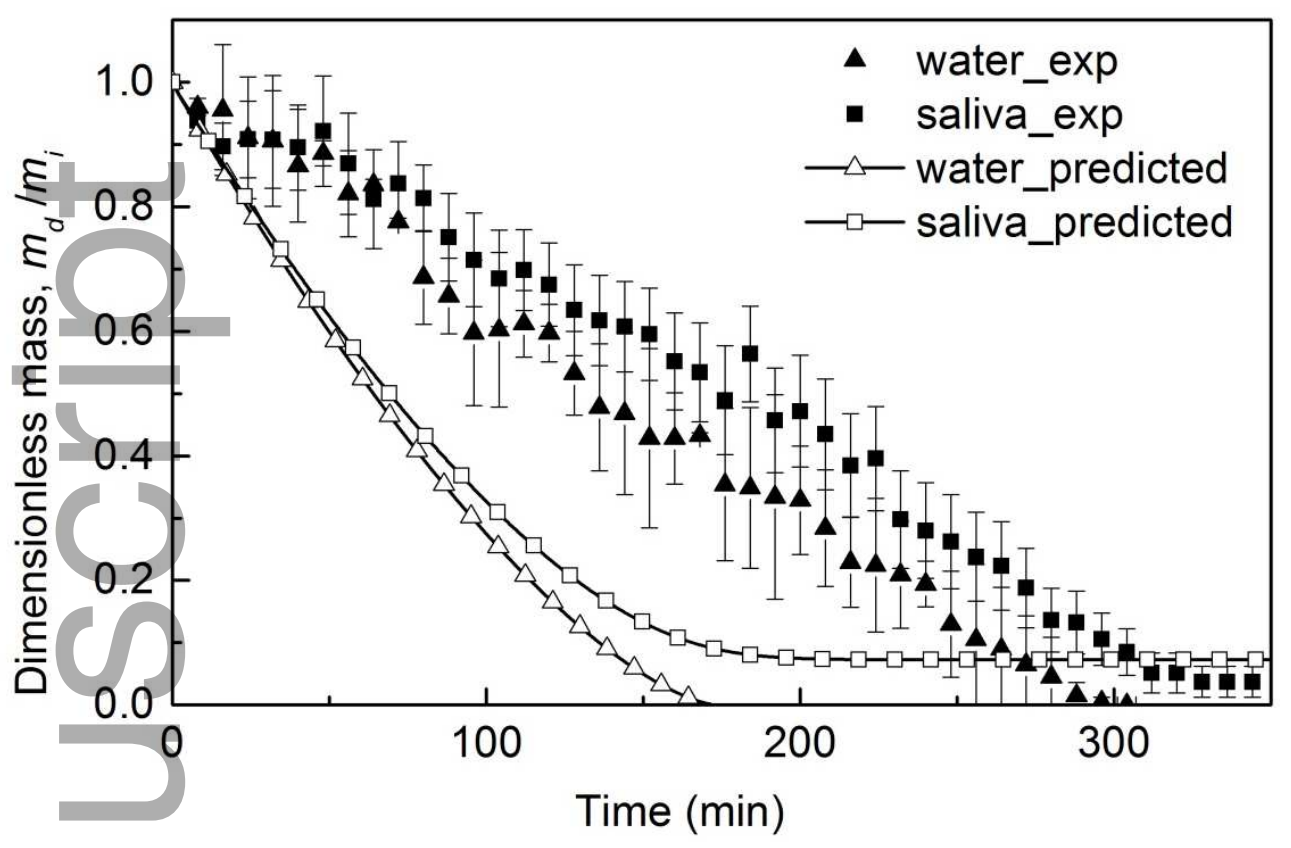

(b)

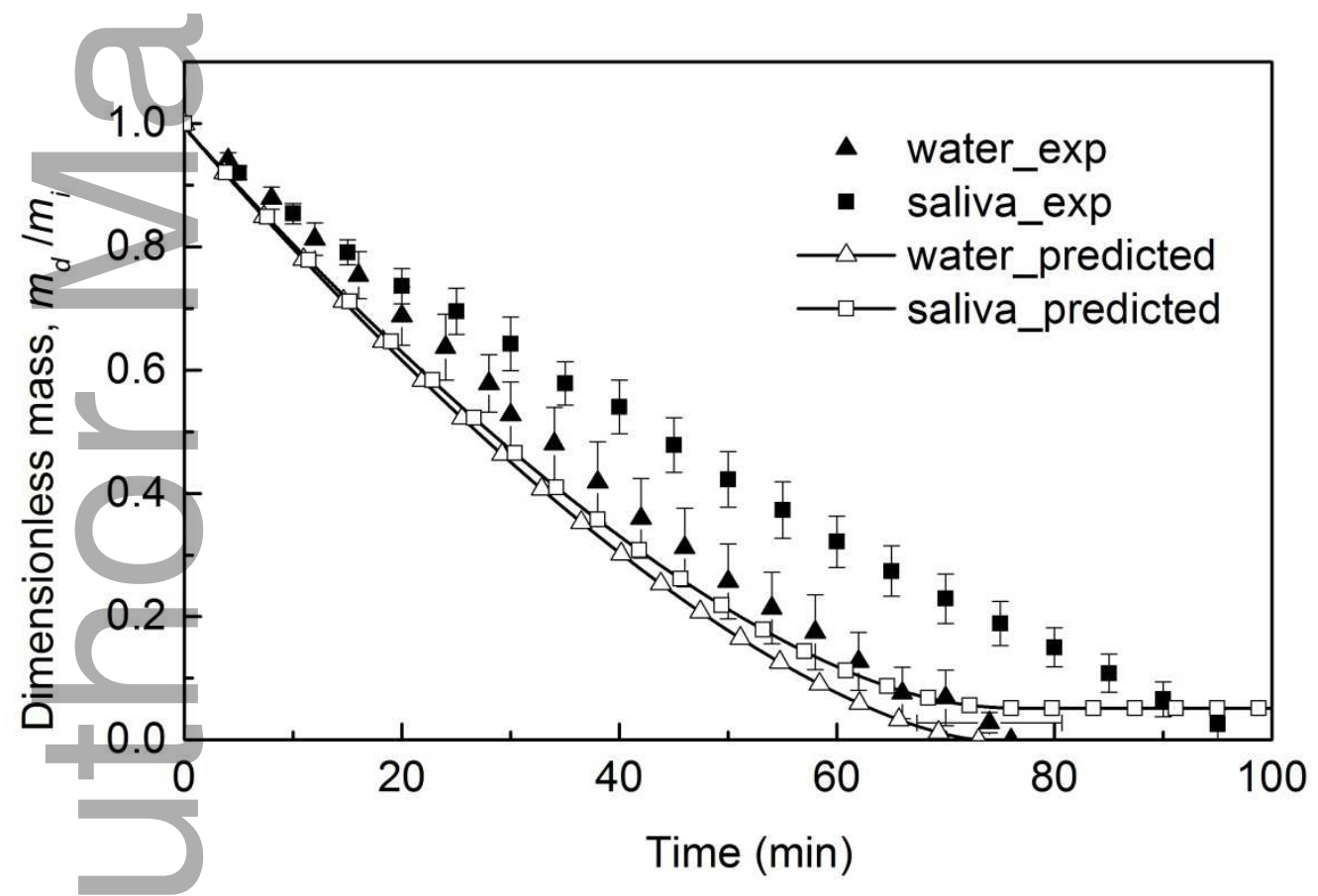

(c)

Figure 4. Mass losses of water droplets and saliva droplets at (a) $\mathrm{RH}=96 \%$, (b) $84 \%$ and (c) $64 \%$. The initial droplet size is about $1337 \mu \mathrm{m}$, and droplet mass $\left(m_{d}\right)$ is normalized by the initial droplet mass $\left(m_{i}\right)$. The experiments were repeated three times, and the error bars refer to the standard error. $\mathrm{RH}$, relative humidity. 


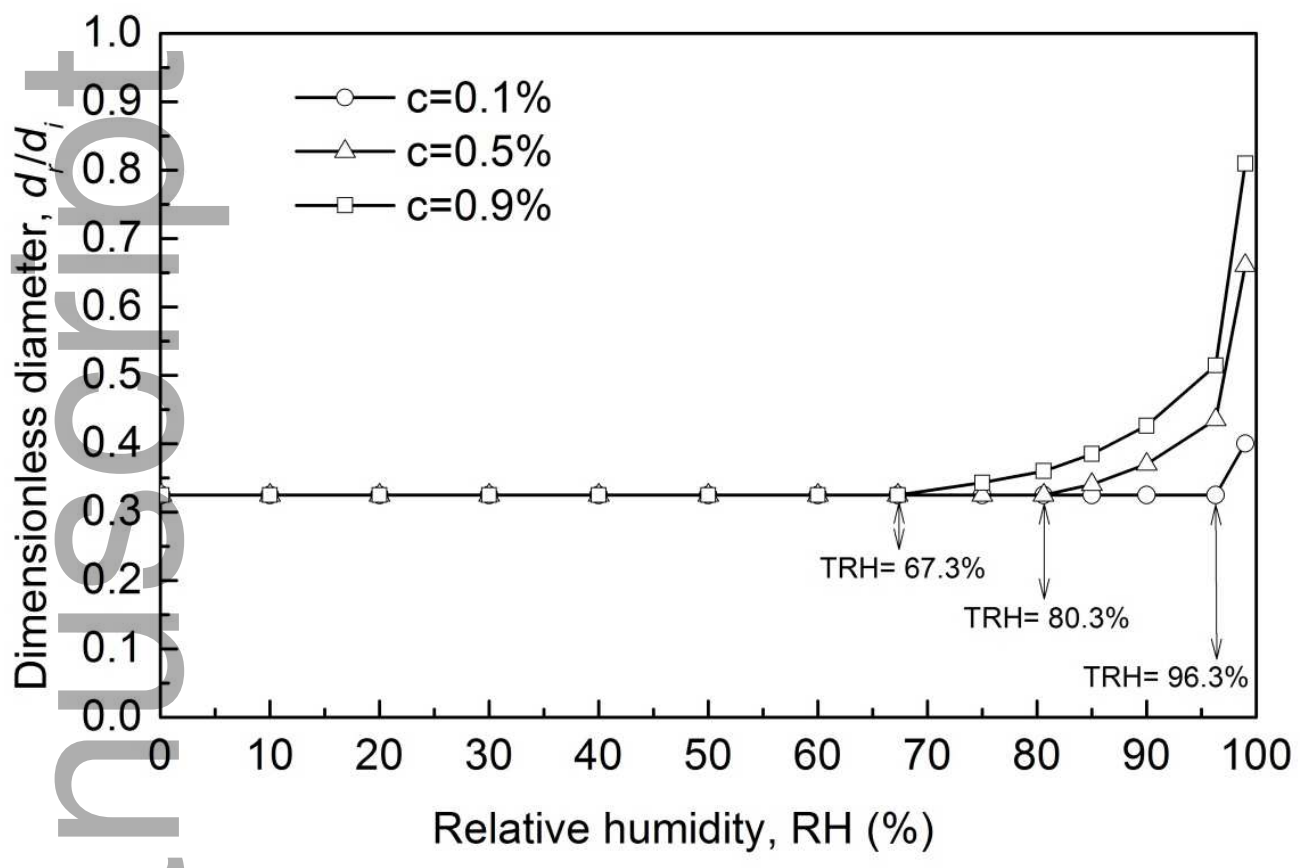

(a)

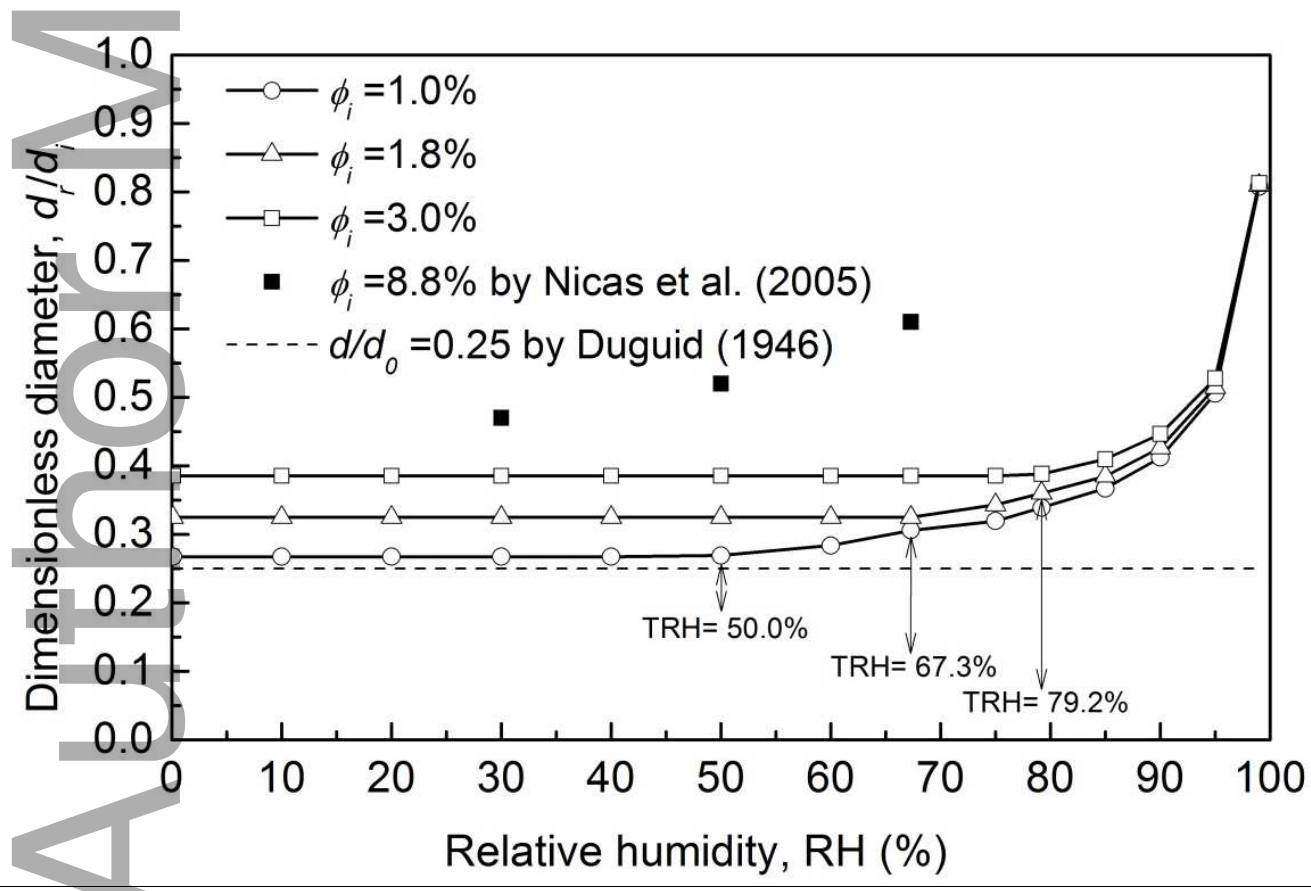

(b)

Figure 5. Dimensionless diameter of the residue size of droplets. (a) Dependence of residue size on initial salt mass fraction $c_{i}\left(\Phi_{i}=1.8 \%\right)$. (b) Dependence of residue size on the initial volume ratio of particles $\Phi_{i}\left(c_{i}=0.9 \%\right)$. $\mathrm{CRH}$, critical relative humidity; TRH, threshold This article is protected by copyright. All rights reserved 
relative humidity.

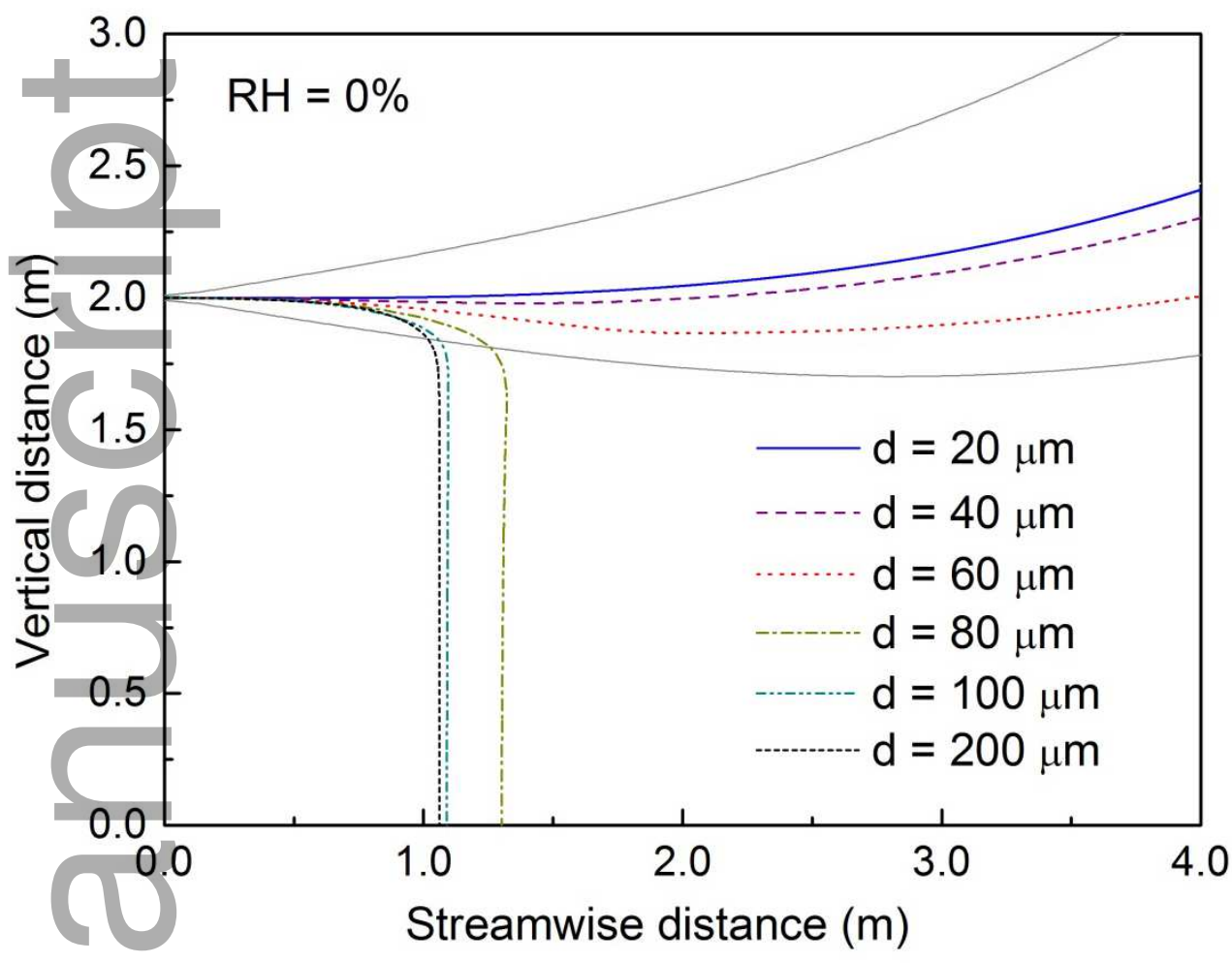

(a)

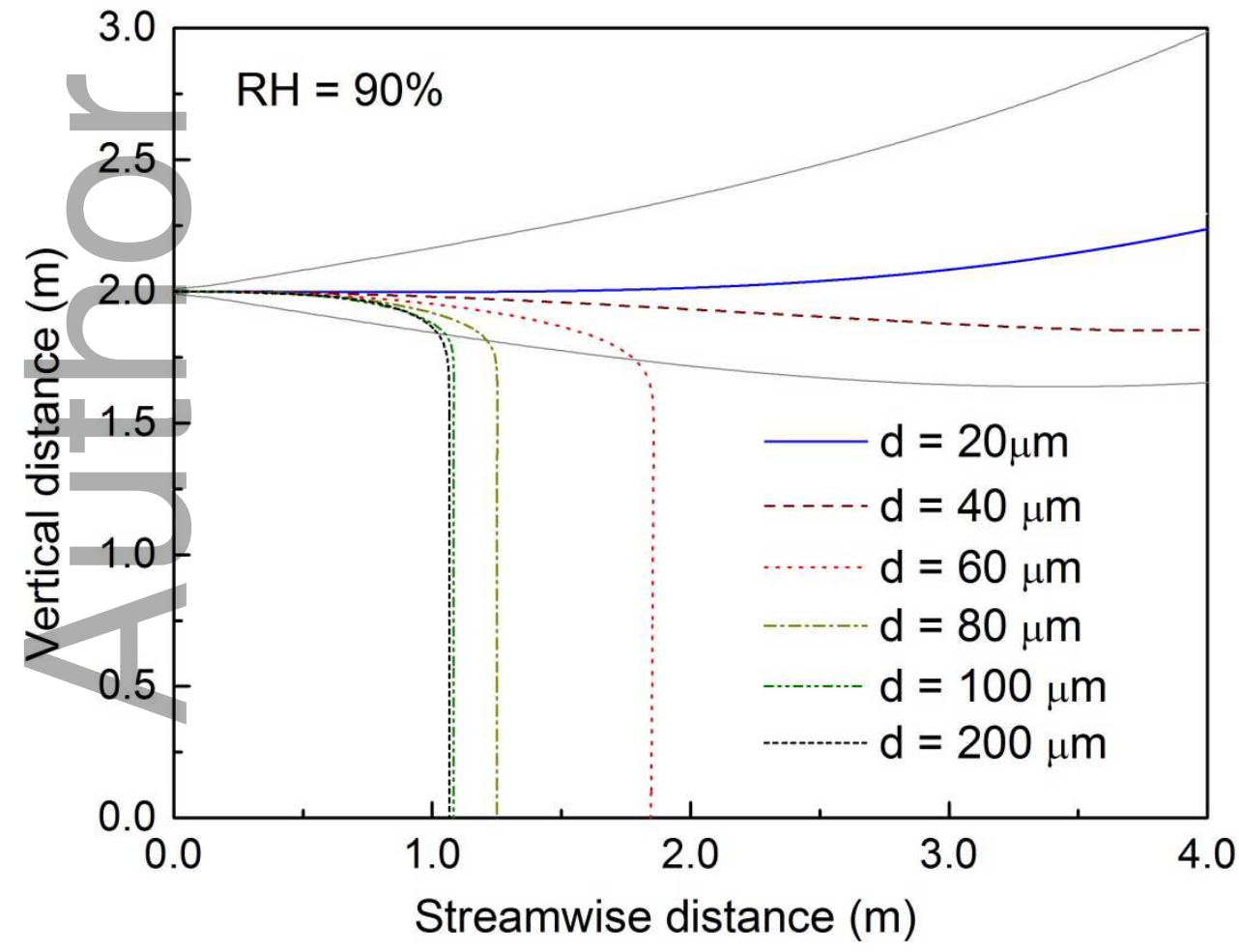

This article is protected by copyright. All rights reserved 
(b)

Figure 6. Trajectories of droplets of different sizes released from a cough jet without turbulence. The initial velocity is $10 \mathrm{~m} / \mathrm{s}$, and the ambient temperature is $25^{\circ} \mathrm{C}$. The jet boundary is indicated by the grey lines. (a) The RH in the surrounding environment is $0 \%$. (b) The $\mathrm{RH}$ in the surrounding environment is $90 \%$. $\mathrm{RH}$, relative humidity.

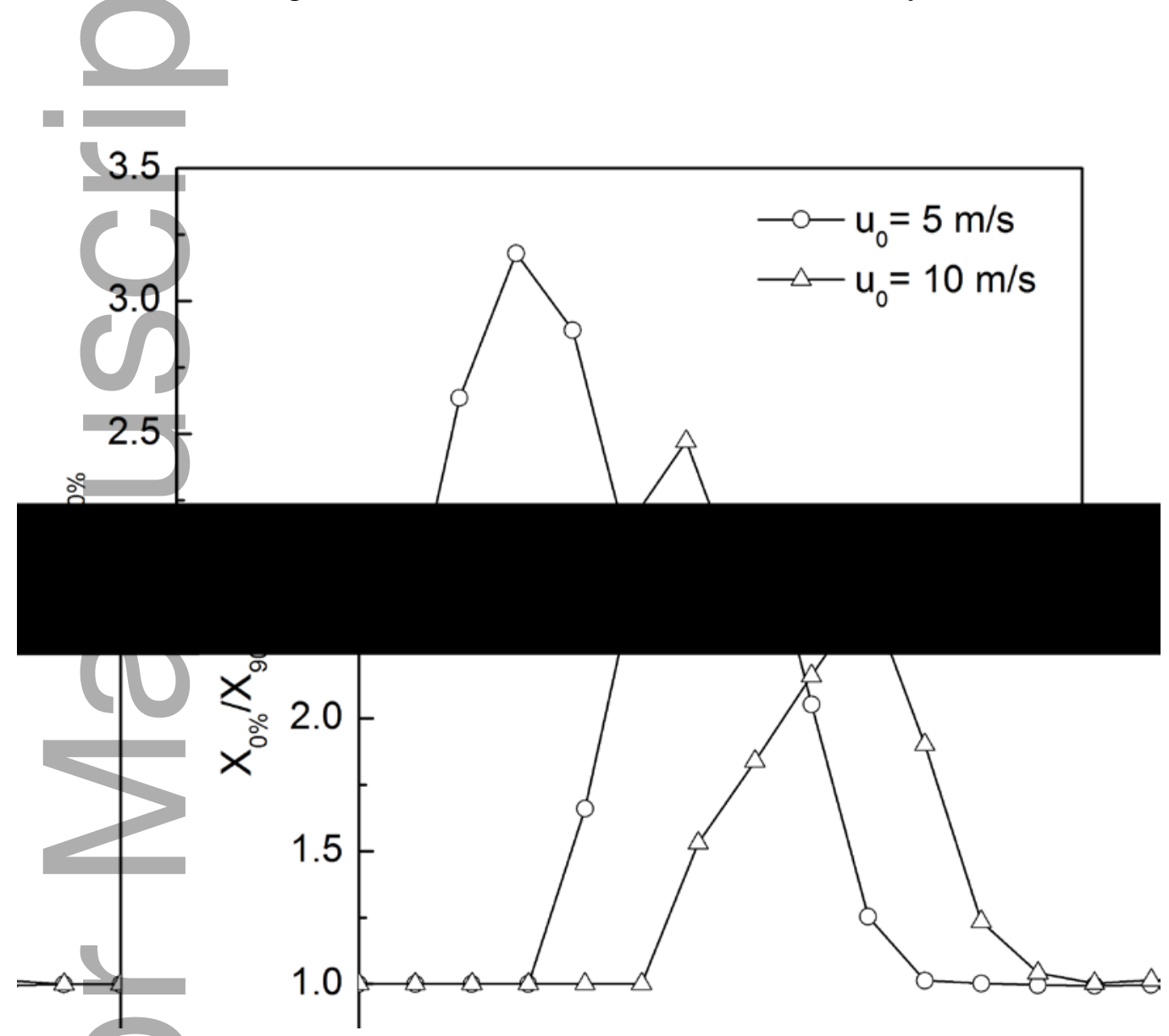

Figure 7. Ratio of the maximum horizontal travel distance for one droplet when $\mathrm{RH}$ is $0 \%$ to the distance when $\mathrm{RH}$ is $90 \%$. $\mathrm{RH}$, relative humidity.

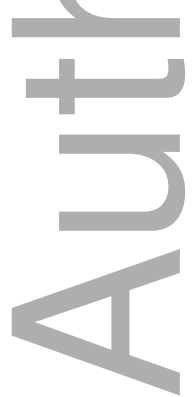



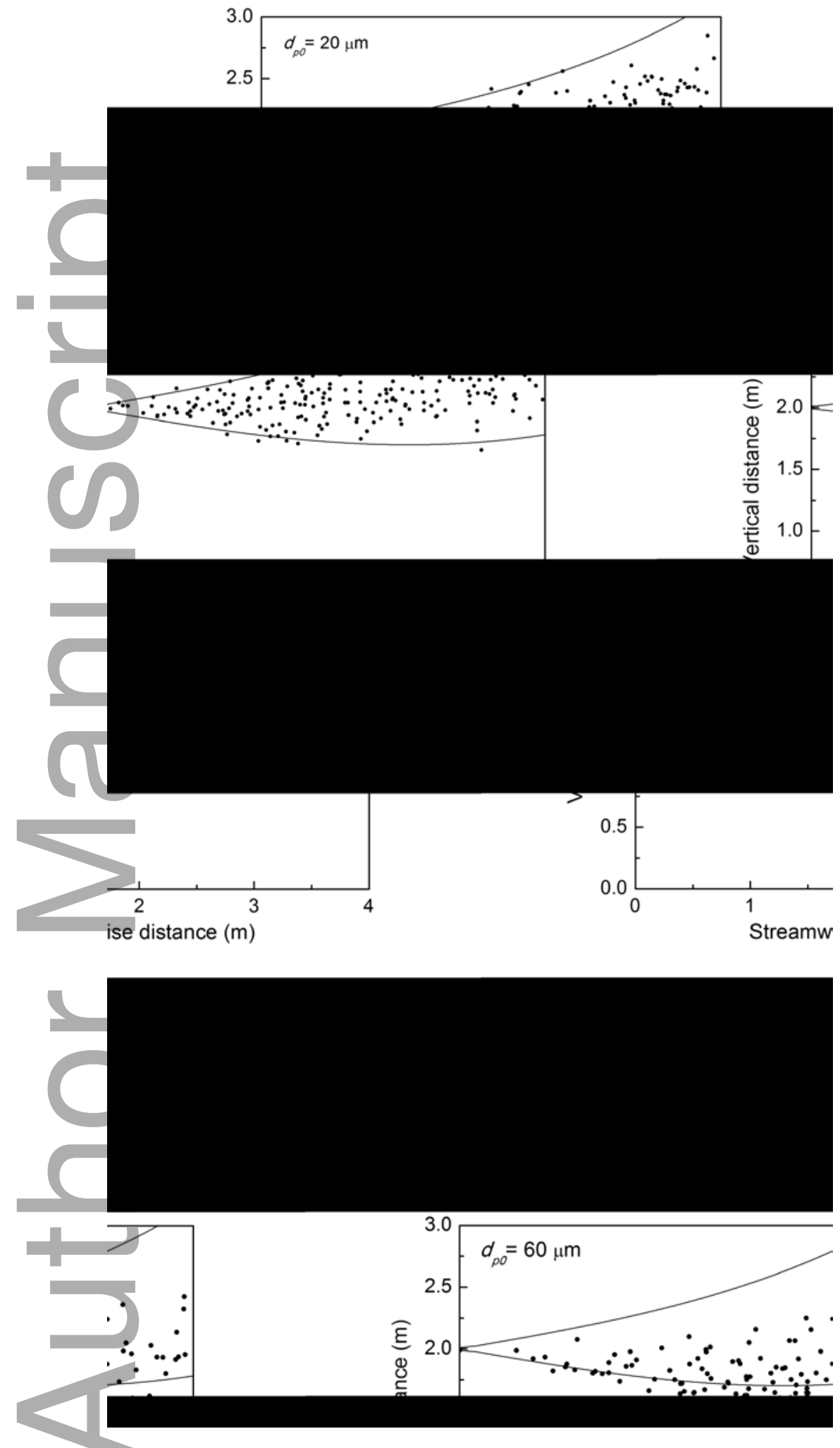

Figure 8. Droplet dispersion in the turbulent buoyant jet $\left(D=2 \mathrm{~cm}, U_{0}=10 \mathrm{~m} / \mathrm{s}, T_{\infty}=25^{\circ} \mathrm{C}\right.$, $\mathrm{RH}=0 \%$ ). The grey lines correspond to the jet boundary. $\mathrm{RH}$, relative humidity. 

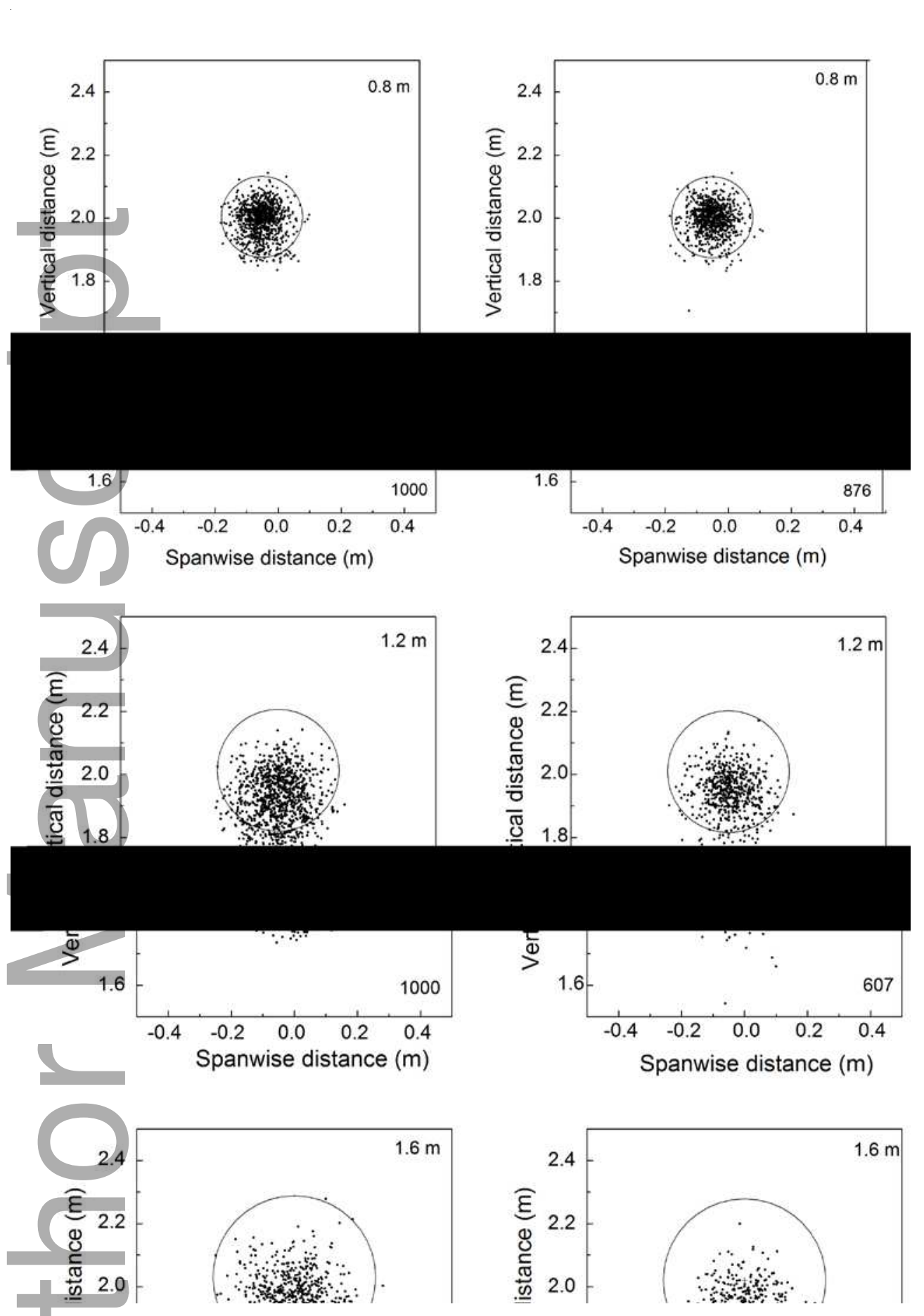

Figure 9. Dispersion of 1000 individually suspended droplets at $0.8,1.2$ and $1.6 \mathrm{~m}$ away from the opening. The droplet initial diameter is $60 \mu \mathrm{m}$, and the jet boundary is indicated by the grey line. Left panels $(\mathrm{RH}=0 \%)$; right panels $(\mathrm{RH}=90 \%)$. The number of remaining particles is shown in the bottom right corner of each panel. RH, relative humidity. 


\section{University Library}

\section{- M M I E E R VA A gateway to Melbourne's research publications}

Minerva Access is the Institutional Repository of The University of Melbourne

Author/s:

Liu, L;Wei, J;Li, Y;Ooi, A

Title:

Evaporation and dispersion of respiratory droplets from coughing

Date:

2017-01-01

Citation:

Liu, L., Wei, J., Li, Y. \& Ooi, A. (2017). Evaporation and dispersion of respiratory droplets from coughing. INDOOR AIR, 27 (1), pp.179-190. https://doi.org/10.1111/ina.12297.

Persistent Link:

http://hdl.handle.net/11343/291093 